\title{
硫代磷(膦)酸酯的合成研究进展
}

\author{
刘春琪 $a$ 王莉贤 $a$ 张兴华*,a,b \\ ( ${ }^{a}$ 上海应用技术大学化学与环境工程学院 上海 201418) \\ ( b 宁波大学新药技术研究院 浙江宁波 315211)
}

\begin{abstract}
摘要 硫代磷(膦)酸酷在农药、医药以及有机合成等研究领域具有广泛应用. 重点总结了由 $\mathrm{P}(\mathrm{O})-\mathrm{H}$ 化合物出发合成 硫代磷(膦)酸酯类化合物的研究方法, 按硫元素的不同来源分类, 对该类反应的研究进展进行综述.

关键词 硫代磷酸酯; 硫代膦酸酯; $\mathrm{P}(\mathrm{O})-\mathrm{H}$ 化合物
\end{abstract}

\section{Advances in the Synthesis of Phosphorothioate and Phosphinothioate}

\author{
Liu, Chunqi ${ }^{a} \quad$ Wang, Lixian ${ }^{a} \quad$ Zhang, Xinghua ${ }^{*, a, b}$ \\ ( ${ }^{a}$ School of Chemical and Environmental Engineering, Shanghai Institute of Technology, Shanghai 201418) \\ ( ${ }^{b}$ Institute of Drug Discovery Technology, Ningbo University, Ningbo, Zhengjiang 315211)
}

\begin{abstract}
Organophosphorus compounds which contain phosphorus-sulfur bonds have been widely used in the fields of pesticides, pharmaceuticals and organic synthesis. The recent progress of the methods for the synthesis of phosphorothioate and phosphinothioate from $\mathrm{P}(\mathrm{O})-\mathrm{H}$ reagents are summarized on the basis of different types of sulfur source.
\end{abstract}

Keywords phosphorothioate; phosphinothioate; $\mathrm{P}(\mathrm{O})-\mathrm{H}$ compound

硫代磷(膦)酸酯由于其特殊的生物活性和理化性质 被广泛应用于医药、农药以及有机合成等领域 $[1-3]$. 医学 上, 在治疗癌症、青光眼、心力衰竭等 $[4-6]$ 疾病中, 含有 $\mathrm{P}(\mathrm{O})-\mathrm{S}$ 键的药物可以起到一定的辅助治疗作用, 此外, 部分硫代磷酸酯类化合物也具有较好的杀虫杀菌活 性 ${ }^{[7-9]}$, 如异稻瘟净 $(O, O$-二异丙基- $S$-芐基硫代磷酸酯) 就是一种在农业上广泛使用的杀菌剂(图 1, F).

传统合成此类化合物的方法通常采用 MichaelisArbuzov 反应(Eq. 1), 以磺酰氯为硫源实现从亚磷酸酯 到硫代磷酸酯的转化 ${ }^{[10]}$; 从 $\mathrm{P}(\mathrm{O})-\mathrm{H}$ 与硫醇(酚)化合物 出发, 遵循 Atherton-Todd 反应规律 ${ }^{[1-12]}$, 也是构建 $\mathrm{P}-$ $S$ 键化合物的重要方法(Eq. 2). 但这两类反应存在着原 子利用率低以及化学量氯化试剂的使用等问题. 近年来 合成该类有机物的新方法层出不穷. 由 $\mathrm{P}(\mathrm{O})-\mathrm{H}$ 试剂出 发合成硫代磷(膦)酸酯的方法逐渐受到关注, 并且得到 了不断的深入研究. 因此, 本综述从 $\mathrm{P}(\mathrm{O})-\mathrm{H}$ 化合物出 发, 根据参与反应的硫元素的不同来源进行划分，依次

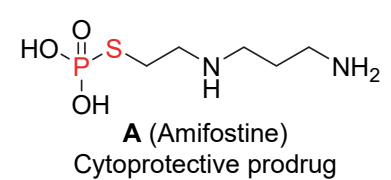<smiles>CCOP(=O)(OCC)SC[C@]1(C)C=C(n2cnc3c(N)nc(I)nc32)[C@H](O)[C@@H]1OC(C)(C)C</smiles><smiles>COP(=O)(OC)SCc1ccccc1</smiles>
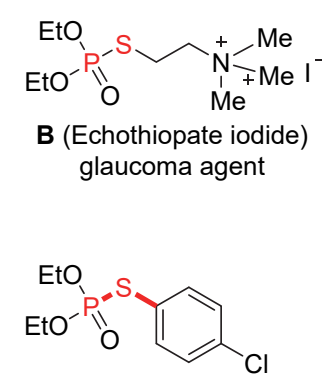

D (Anticholinesterases)

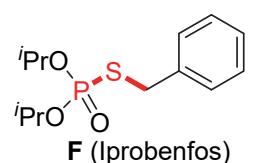

图 1 硫代磷酸酯在医药、农药方面的应用

Figure 1 Application of phosphorothioate in medicine and pesticides

按照硫醇(酚)、双硫化合物、磺酰类化合物、硫单质以

* Corresponding author. E-mail: xhzhang@sit.edu.cn

Received April 1, 2021; revised May 6, 2021; published online May 24, 2021.

Project supported by the National Natural Science Foundation of China (No. 21871182).

国家自然科学基金(No. 21871182)资助项目. 
及其他含硫试剂的顺序, 对近年来硫代磷(膦)酸酯的合 成方法进行归纳总结.

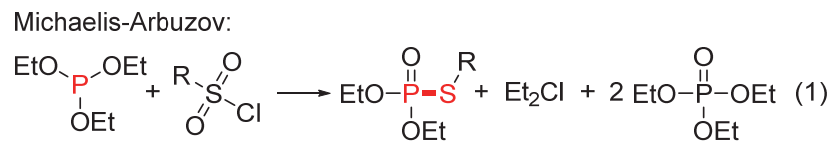

Atherton-Todd:

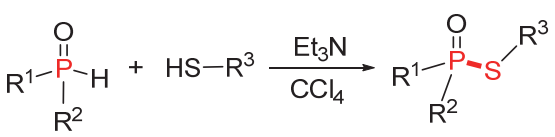

\section{$1 \mathrm{P}(\mathrm{O})$ - H 与硫醇/酚交叉偶联构建硫代磷(膦)}

\section{酸酯}

\section{1 过渡金属催化合成硫代磷(膦)酸酯}

2013 年, Kaboudin 课题组 ${ }^{[13]}$ 报道了碘化亚铜催化合 成硫代磷酸酯类化合物的方法(Eq. 3). 该方法操作简 便、条件温和, 且有较好收率. 但对于底物磷试剂的种 类具有局限性，只适用于烷氧基磷酸酯，不适用于含芳 基的磷酸酯.

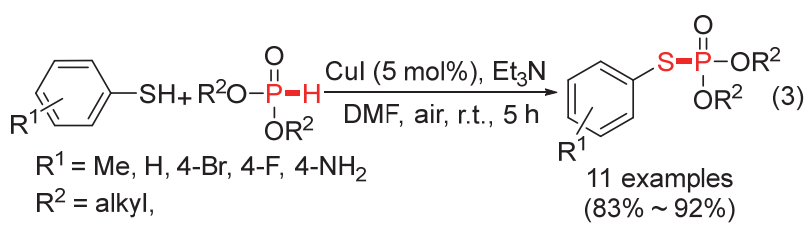

2016 年, 韩立彪等 ${ }^{[14]}$ 开发了一种钯催化硫醇(酚)与 $\mathrm{P}(\mathrm{O})-\mathrm{H}$ 化合物氧化脱氢偶联 $(\mathrm{CDC})$ 合成硫代磷酸酯的 方法(Eq. 4). 该方法避免了传统氯化试剂 $\mathrm{CCl}_{4}$ 的使用, 具有良好的底物普适性, 对于手性磷试剂在内的多种有 机磷试剂(H-膦酸盐, 次磷酸盐和仲膦氧化物)均适用. 在机理研究中发现, 该反应利用 $\mathrm{Pd}$ 的自身变价来实现 氧化还原.

2018 年, Kang 等 ${ }^{[15]}$ 开发了一种酞菁铁 $[\mathrm{Fe}(\mathrm{Pc})]$ 催化 硫醇与 $\mathrm{P}(\mathrm{O})$ - $\mathrm{H}$ 试剂交叉脱氢偶联构建 $\mathrm{S}-\mathrm{P}(\mathrm{O})$ 键化合 物的新方法(Eq. 5). 作者在机理研究中利用加入自由基 捕获剂[四甲基哌啶氧化物(TEMPO)、2,6-二叔丁基-4甲基苯酚(BHT)], 推测该反应属于自由基反应.

\section{2 碱促进硫代磷(膦)酸酯的合成}

2015 年, 韩立彪课题组 ${ }^{[16]}$ 利用叔丁醇锂作为碱, 合 成了一系列硫代膦酸酯(Eq. 6). 该方法具有较好的底物 适应性, 不仅适用于硫酚, 在反应中氯仿作为氯化试剂 以及反应溶剂, 避免了传统 Atherton-Todd 反应中 $\mathrm{CCl}_{4}$ 的使用, 也实现了硫醇作为底物, 同时该方法对于芳香 醇以及苯硒酚也有较好的底物适用性.

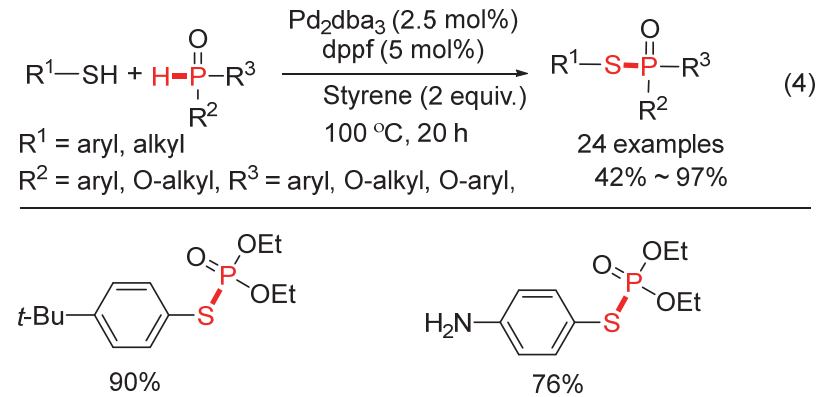<smiles>CCOP(=O)(OCC)Sc1ccc(O)cc1</smiles>

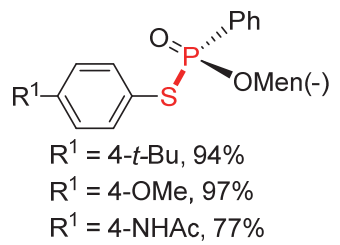<smiles>CC(C)(C)c1ccc(SP2(=O)Oc3ccccc3-c3ccccc32)cc1</smiles>
$59 \%$

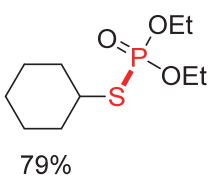

$79 \%$

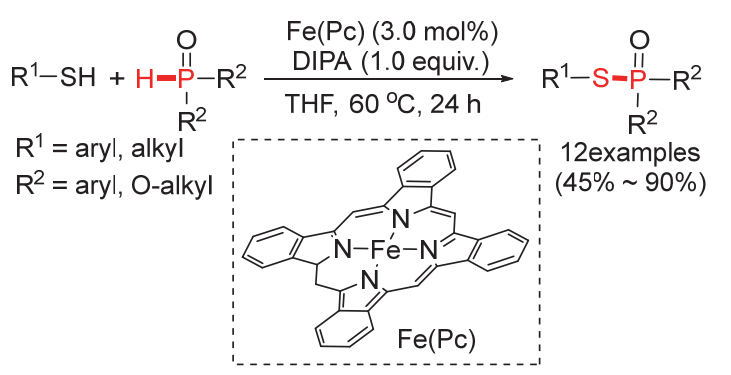

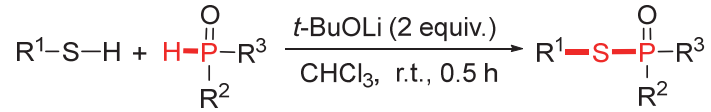

$$
\begin{aligned}
& \mathrm{R}^{1}=\text { aryl, alkyl } \\
& \mathrm{R}^{2}, \mathrm{R}^{3}=\text { phenyl, aryl, alkyl }
\end{aligned}
$$

2017 年, 焦宁课题组 ${ }^{[17]}$ 报道了 $\mathrm{Cs}_{2} \mathrm{CO}_{3}$ 调节 $\mathrm{P}(\mathrm{O})-$ $\mathrm{H}$ 化合物与硫醇(酚)脱氢偶联制备硫代磷酸酯的方法 (Eq. 7). 该反应以氧气作为氧化剂, 具有反应条件温和, 官能团耐受性好等特点(Scheme 2), 不仅可以实现模型 反应的克级制备, 而且能以较好收率合成一系列生物活 性分子. 作者为解释氧气的氧化作用, 利用对比实验阐 明了反应的关键步骤是双硫化合物的形成，再由双硫化 合物到最终的硫代磷酸酯.

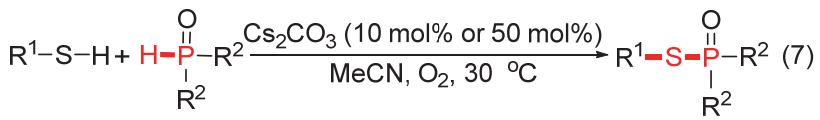

$$
\begin{aligned}
& \mathrm{R}^{1}=\text { alkyl, aryl } \mathrm{R}^{2}=\text { alkoxyl, aryl } \quad 32 \text { examples } \\
& \text { (22\% 94\%) }
\end{aligned}
$$

同年，王忠卫等 ${ }^{[18]}$ 发展了 $\mathrm{Na}_{2} \mathrm{CO}_{3}$ 促进 $\mathrm{P}(\mathrm{O})-\mathrm{S}$ 化 合物的合成(Eq. 8). 该方法以 $\mathrm{Na}_{2} \mathrm{CO}_{3}$ 作为碱, 在室温下 


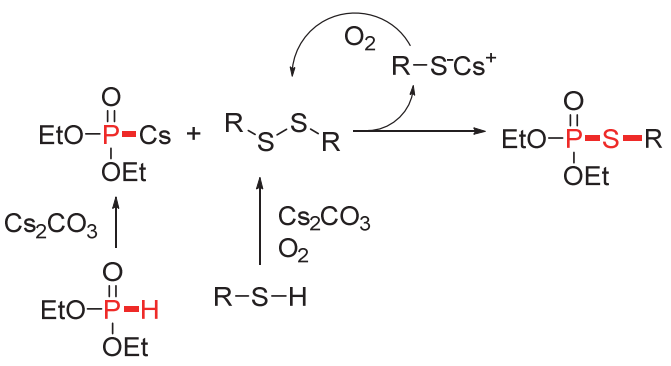

图式 $2 \mathrm{Cs}_{2} \mathrm{CO}_{3}$ 调节的偶联反应

Scheme $2 \mathrm{Cs}_{2} \mathrm{CO}_{3}$ promoted coupling reaction

空气中即可实现 $\mathrm{P}(\mathrm{O})-\mathrm{S}$ 键的构建, 但产物主要为芳基 硫代膦酸酯。

$$
\begin{aligned}
& \mathrm{R}^{1}-\mathrm{SH}+\underset{\mathrm{R}^{2}}{\stackrel{\mathrm{H}-\mathrm{P}_{1}^{\mathrm{O}}}{\mathrm{I}}-\mathrm{R}^{3}} \frac{\mathrm{Na}_{2} \mathrm{CO}_{3} \text { (equiv.) }}{\mathrm{DMF} \text {, air, r.t., } 3 \mathrm{~h}} \mathrm{R}^{1}-\mathrm{S}-\underset{\mathrm{P}_{1}}{\mathrm{O}}-\mathrm{R}^{3} \\
& \mathrm{R}^{1}=\text { aryl } \mathrm{R}^{2}, \mathrm{R}^{3}=\text { phenyl, aryl, alkoxyl } \quad 22 \text { examples }
\end{aligned}
$$

\section{3 氧化剂促进硫代磷(膦)酸酯的合成}

2015 年, 潘远江等 ${ }^{[19]}$ 报道了在碘化钾 $(\mathrm{KI})$ 和过氧化 苯甲酸特丁酯(TBPB)的协同作用下, $\mathrm{P}(\mathrm{O})-\mathrm{H}$ 化合物与 硫醇/酚偶联构建 $\mathrm{P}(\mathrm{O})-\mathrm{S}$ 键的方法(Eq. 9). 该反应具有 较好的普适性, 在无酸碱与过渡金属参与的条件下即可 实现相应硫代磷酸酯的合成. 作者通过自由基跟踪实验 确定该反应为自由基反应，并提出了合理的反应机制 (Scheme 3). 作者推测由 TBPB 在 KI 原位激发形成碘自 由基和颈基自由基 $\mathbf{A}$, 同时烷氧自由基与磷试剂脱氢产 生磷酰自由基 $\mathbf{B}$, 并迅速与颈基自由基 $\mathbf{A}$ 偶联得到最终 产物.

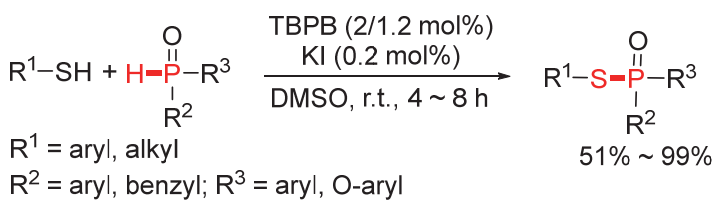

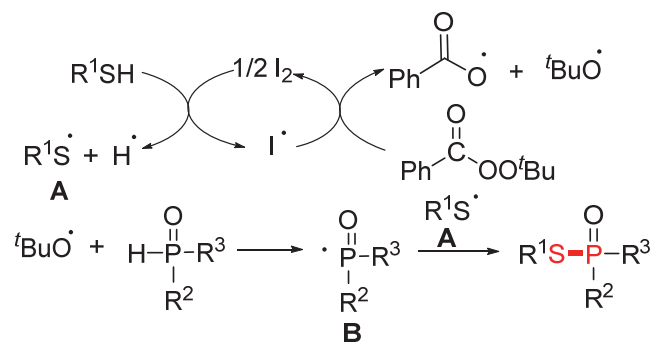

图式 $3 \mathrm{KI} / \mathrm{TBPB}$ 促进硫代膦酸酯化合物的制备 Scheme 3 KI/TBPB promotes the preparation of thiophosphonate compounds

随后，潘远江等 ${ }^{[20]}$ 又发展了二叔丁基过氧化物 (DTBP) 促进的 $\mathrm{P}(\mathrm{O})-\mathrm{H}$ 化合物与硫酚脱氢偶联的方法 (Eq. 10). 该方法克服了 TBPB 催化的磷试剂只适用于芳
香基团的局限性，多官能团底物的拓展性广，产率为 $27 \% \sim 97 \%$.

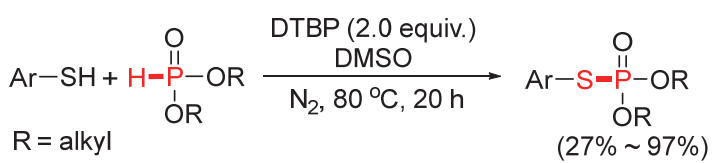

2015 年，肖军华等[21]发现强氧化剂 1,3-二氯-5,5-二 甲基海因(DCDMH), 可以实现硫代磷酸酯的高效制备 (Eq. 11). 该反应避免了金属和其他添加剂的参与，条件 温和，反应时间短，产率较高，但由于强氧化剂的作用， 生成副产物二硫化物，降低了原子利用率。另外，芳基 硫醇相对脂肪族硫醇的官能团耐受性较好.

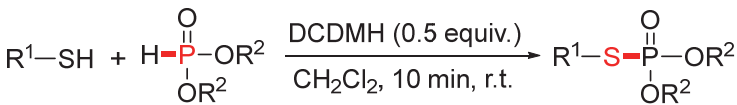

$$
\begin{aligned}
& \mathrm{R}^{1}=\text { alkyl, aryl } \mathrm{R}^{2}=\text { alkyl } \\
& \text { DCDMH }
\end{aligned}
$$

2017 年，张博等 ${ }^{[22]}$ 利用二甲亚砜(DMSO)作为氧剂, 实现了苯硫酚(双硫醚)与 $\mathrm{P}(\mathrm{O})-\mathrm{H}$ 化合物的直接偶联合 成硫代膦(磷)酸酯(Eq. 12). DMSO 较前文提及的氧化剂 (TBPB、DTBP、DCDMH 等)更温和、便宜、易得，作 者推测的反应机制如 Scheme 4 所示, 通过机理研究发 现，反应中重要的一步是硫酚在 DMSO 作用下形成双 硫化物的中间体.

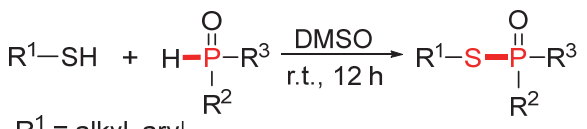

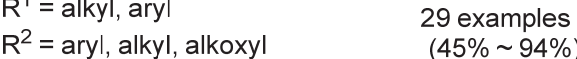

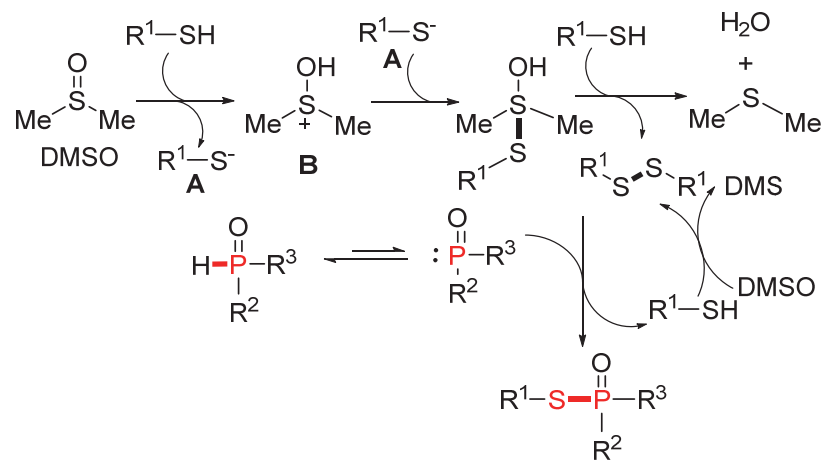

图式 4 DMSO 调节的偶联反应

Scheme 4 DMSO promoted coupling reaction

\section{4 光/电催化合成硫代磷(膦)酸酯}

光催化作为有机合成中的一种绿色合成手段, 在催 化合成硫代磷(膦)酸酯方面也得到了很好的利用. 2016 
年，张博等 ${ }^{[23]}$ 报道了光催化下硫酚与 $\mathrm{P}(\mathrm{O})$ - $\mathrm{H}$ 化合物构 建 $\mathrm{P}(\mathrm{O})-\mathrm{S}$ 键的方法(Eq. 13). 该反应条件温和无需金属 参与, 以玫瑰红作为光催化剂实现了 $\mathrm{P}(\mathrm{O})-\mathrm{H}$ 化合物与 硫酚的交叉偶联, 副产物仅为水. 有较好的化学选择性 和官能团的耐受性, 并可以实现克级制备.

作者对机理进行研究，提出了合理的反应机制：首 先玫瑰红在可见光催化下与氧气反应产生单线态氧, 然 后单线态氧分别与 $\mathrm{P}(\mathrm{O})-\mathrm{H}$ 化合物、硫醇作用得到相应 磷自由基与硫自由基, 自由基可在反应体系中通过三种 不同路径得到最终产物(Scheme 5).

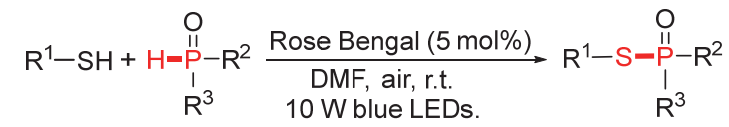

$$
\begin{aligned}
& \mathrm{R}^{1}=\text { alkyl, aryl, benzyl; } \\
& \mathrm{R}^{2}=\text { aryl; } \mathrm{R}^{3}=\text { aryl, alkyl, alkoxyl }
\end{aligned}
$$

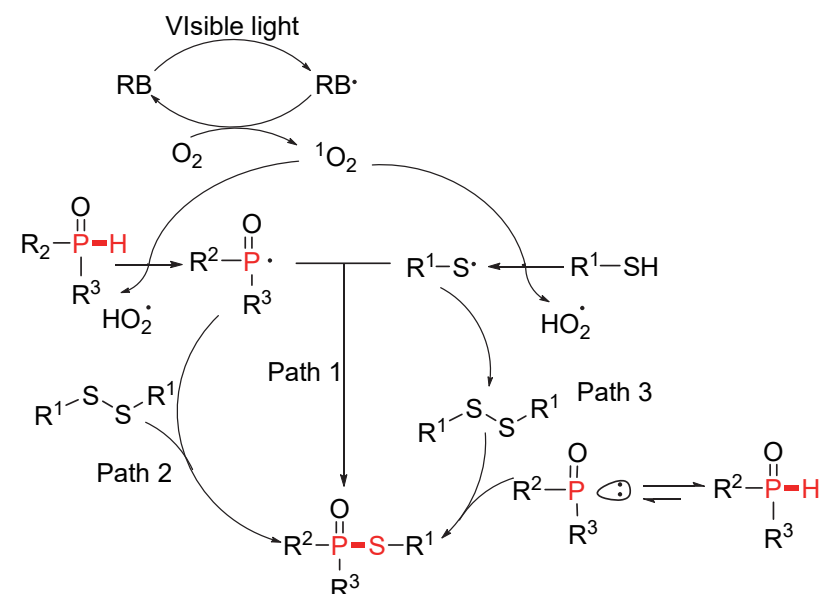

图式 5 光催化交叉偶联反应构建 $\mathrm{P}(\mathrm{O})-\mathrm{S}$ 键

Scheme 5 Photocatalytic cross-coupling reaction to construct $\mathrm{P}(\mathrm{O})-\mathrm{S}$ bond

2018 年, 吴勇等 ${ }^{[24]}$ 利用亚甲基蓝作为光引发剂, 在 碱存在下，通过氧化脱氢偶联反应合成了一系列 $S$-芳基 硫代(膦)磷酸酯(Eq. 14). 该方法不涉及金属及添加剂, 条件温和, 底物适用性更广, 极大地拓展了光催化中 $\mathrm{P}(\mathrm{O})-\mathrm{H}$ 试剂的种类.

在电解催化作用下, 促使硫醇(酚)与 $\mathrm{P}(\mathrm{O})$ - $\mathrm{H}$ 化合 物的交叉偶联也是构建 $\mathrm{P}(\mathrm{O})-\mathrm{S}$ 键的一种绿色途径. 2019 年, 郑柯小组 ${ }^{[25]}$ 利用电极电解的方法实现了二苯 基膦酸与硫醇(酚)的交叉偶联(Eq. 15), 该反应条件温 和, 底物适用性广, 同时也可以实现十克以上级别反应, 具有较好的实用价值.

\section{2 与双硫化合物交叉偶联合成硫代磷(膦)酸酯}

\section{1 利用电解合成硫代磷(膦)酸酯}

1979 年, Torii 等 ${ }^{[26]}$ 报道了在 $\mathrm{NaBr}$ 电解作用下,

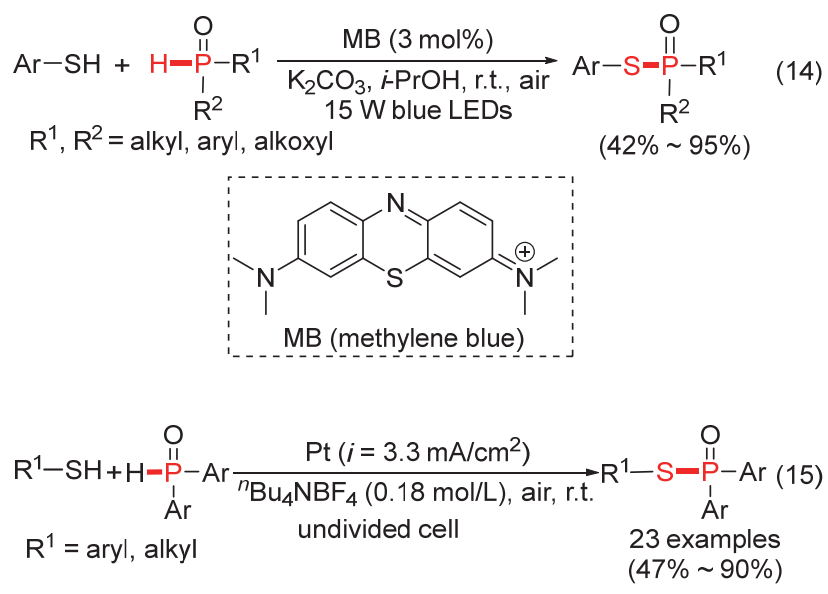

$\mathrm{P}(\mathrm{O})$ - $\mathrm{H}$ 与二硫化物通过交叉偶联反应合成了硫代磷酸 酯的反应(Eq. 16). 此方法具有较高的区域选择和原子 利用率，副产物为 $\mathrm{H}_{2}$.

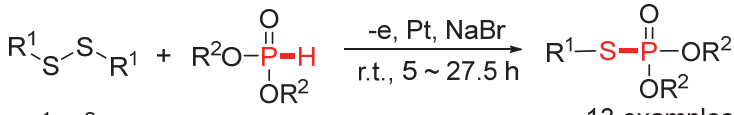

$$
\begin{aligned}
& \mathrm{R}^{1}, \mathrm{R}^{2}=\text { alkyl, aryl } \\
& 13 \text { examples }
\end{aligned}
$$

作者对反应机理做了推测(Scheme 6). 在电解作用 下，溴离子在阳极失去电子发生氧化反应得到溴单质， 立即与一分子亚磷酸酯结合得到溴代膦酸酯, 澳代磷酸 酯与二硫化物作用生成目标产物以及亚磺酰基澳化物. 随后, 亚磺酰基溴化物与亚磷酸酯也可以交叉偶联生成 了硫代磷酸酯并释放出澳化氢，最终溴化氢与体系中的 电子结合释放出氢气与澳离子, 溴离子将继续参与到反 应循环中。

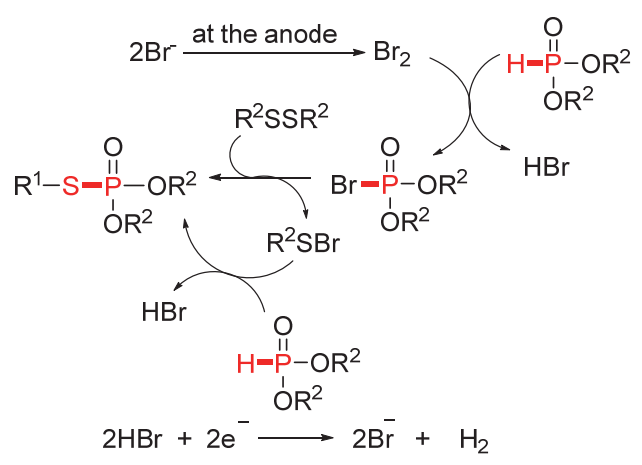

图式 $6 \mathrm{P}(\mathrm{O})-\mathrm{H}$ 与二硫化物的直接偶联

Scheme 6 Direct coupling of $\mathrm{P}(\mathrm{O})-\mathrm{H}$ to disulfides

\section{2 过渡金属催化合成硫代磷(膦)酸酯}

2009 年, 赵玉芬课题组 ${ }^{[27]}$ 在研究二烷基亚磷酸酯 与二芳基二硫醚的氧化交叉偶联反应的过程中发现，在 硒化亚铜的催化作用下，可以实现 $\mathrm{P}(\mathrm{O})-\mathrm{S}$ 键的构建 (Eq. 17). 此外, 该方法同样适用于底物双硒试剂或双锑 
试剂, 首次实现了含 $\mathrm{P}(\mathrm{O})-\mathrm{S}$ 键、 $\mathrm{P}(\mathrm{O})-\mathrm{Se}$ 键、 $\mathrm{P}(\mathrm{O})$ $\mathrm{Te}$ 键化合物的系列合成. 该方法不仅条件温和, 而且对 空气和水分不敏感.

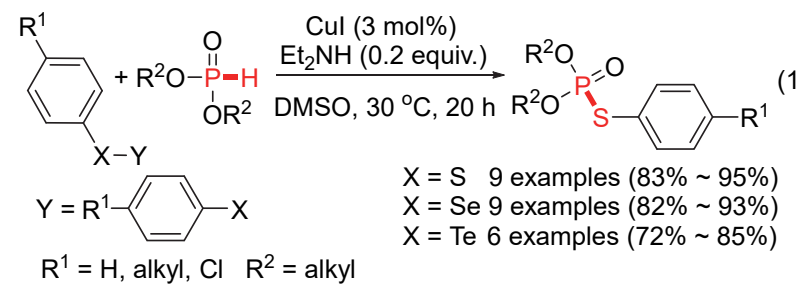

\section{3 碱催化合成硫代磷(膦)酸酯}

2013 年, 许新华课题组 ${ }^{[28]}$ 报道了室温条件下二烷 基磷酸酯与芳基二硫化物的偶联反应(Eq. 18). 该方法 以氢氧化铯 $(\mathrm{CsOH})$ 为催化剂, 实现了多种硫代磷酸酯 的高效合成. 对反应机理也提出了较为合理的推断, 即 氢氧化铯促进下中间体 $(\mathrm{RO})_{2} \mathrm{P}^{-}(\mathrm{O}) \mathrm{Cs}^{+}$的形成以及其与 双硫化物的亲核取代反应对 $\mathrm{P}(\mathrm{O})-\mathrm{S}$ 键的构建起到了决 定性作用(Scheme 7).

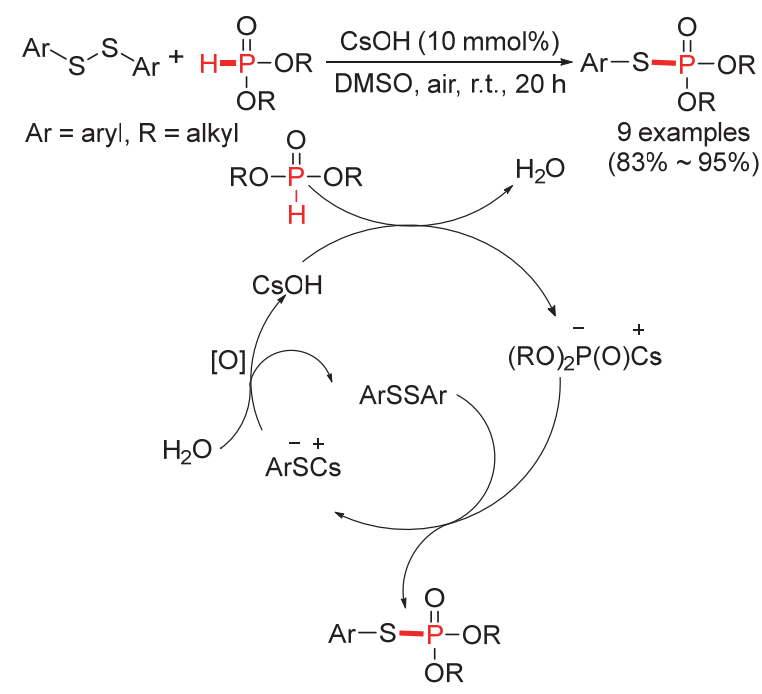

图式 $7 \mathrm{CsOH}$ 催化氢亚磷酸酯与二硫化物的偶联反应 Scheme 7 Coupling reaction of $\mathrm{CsOH}$ catalyzed hydrogen phosphite with disulfide

在此基础上, 2015 年, 许新华等 ${ }^{[29]}$ 发展了一种杯 [4] 芳烃 1 协同作用下氢氧化钾促进的 $\mathrm{P}(\mathrm{O})-\mathrm{S}$ 键的合成方 法(Eq. 19). 实验结果表明杯[4]芳烃 1 的使用极大地促 进了反应的进行, 并且在循环使用 6 次后仍能以较优收 率获得目标产物.

\section{4 其他方法合成硫代磷(膦)酸酯}

2018 年, 严捷等 ${ }^{[30]}$ 开发了碳酸铯存在下 $\mathrm{I}_{2}$ 促进合 成系列硫族磷酸酯的方法(Eq. 20). 该方法不需要金属 参与, 条件相对温和, 操作简便, 同时扩展了 $\mathrm{I}_{2}$ 单质在 有机合成中的应用.

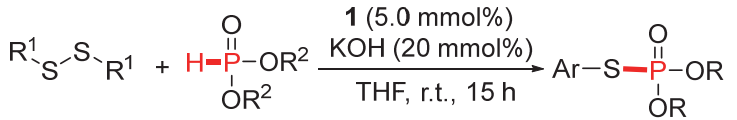

$$
\begin{aligned}
& \mathrm{R}^{1}=\text { aryl, alkyl; } \mathrm{R}^{2}=\text { alkyl } \\
& 13 \text { examples } \\
& \text { (1) } \\
& (85 \% \text { 93\%) }
\end{aligned}
$$

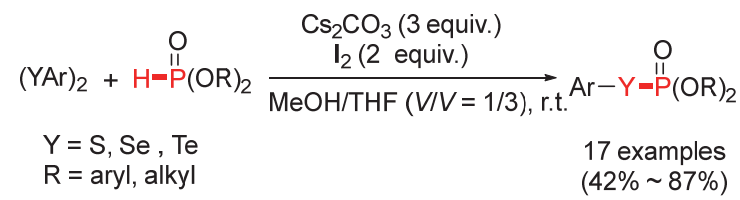

2019年, Badsara 小组 ${ }^{[31]}$ 利用硅胶粉作为媒介, 不使 用催化剂和溶剂, 在室温下实现了双硫试剂与苯基膦酸 的交叉偶联(Eq. 21). 同样的，该方法也能以较优的产率 得到硒(锑)代膦酸酯, 作者对反应机理进行了研究, 推 测该反应为自由基反应.

$$
\begin{array}{ccc}
(\mathrm{SR})_{2}+\mathrm{H}-{ }_{\mathrm{P}}^{\mathrm{O}}(\mathrm{Ar})_{2} & \text { Air } & \stackrel{\mathrm{O}}{\mathrm{N}} \\
\cline { 3 - 3 }=\text { aryl, alkyl } & \text { Silica gel, r.t., } 6 \mathrm{~h} & 18 \text { examples } \\
& & (46 \% \sim 99 \%)
\end{array}
$$

\section{3 与磺酸类化合物交叉偶联合成硫代磷(膦)酸 酯}

\section{1 过渡金属催化合成硫代磷(膦)酸酯}

2014 年, Kumaraswamy 课题组 ${ }^{[32]}$ 报道了碘化亚铜 催化 $\mathrm{P}(\mathrm{O})-\mathrm{H}$ 化合物与磺酰肼氧化脱氢偶联的方法(Eq. 22). 该方法操作简单, 在较短时间内能以较好产率得 到硫代膦酸酯，具有较好的底物普适性，也适用于手性 磷试剂，但在反应过程中手性构型发生了翻转。

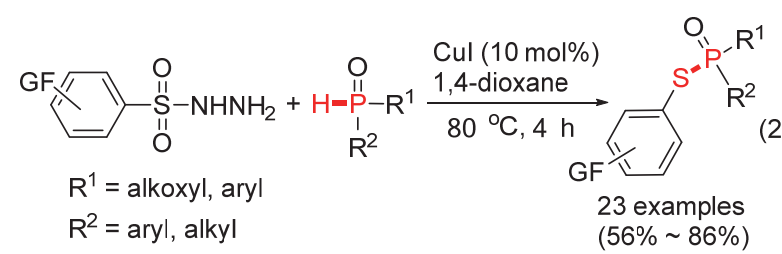

2014 年，吴养洁等 ${ }^{[33]}$ 报道了采用芳基磺酰氯作为 含硫试剂直接合成硫代磷酸酯的方法(Eq. 23). 虽然该 方法具有一定的局限性，如高温、反应时间长、原子经 济性差、产率较低等. 但是，它突破了传统合成方法中 磺酰卤化物不能作为反应底物的难题。作者对反应机理 
进行了研究, 推测其经历了铜变价参与的自由基过程 (Scheme 8).
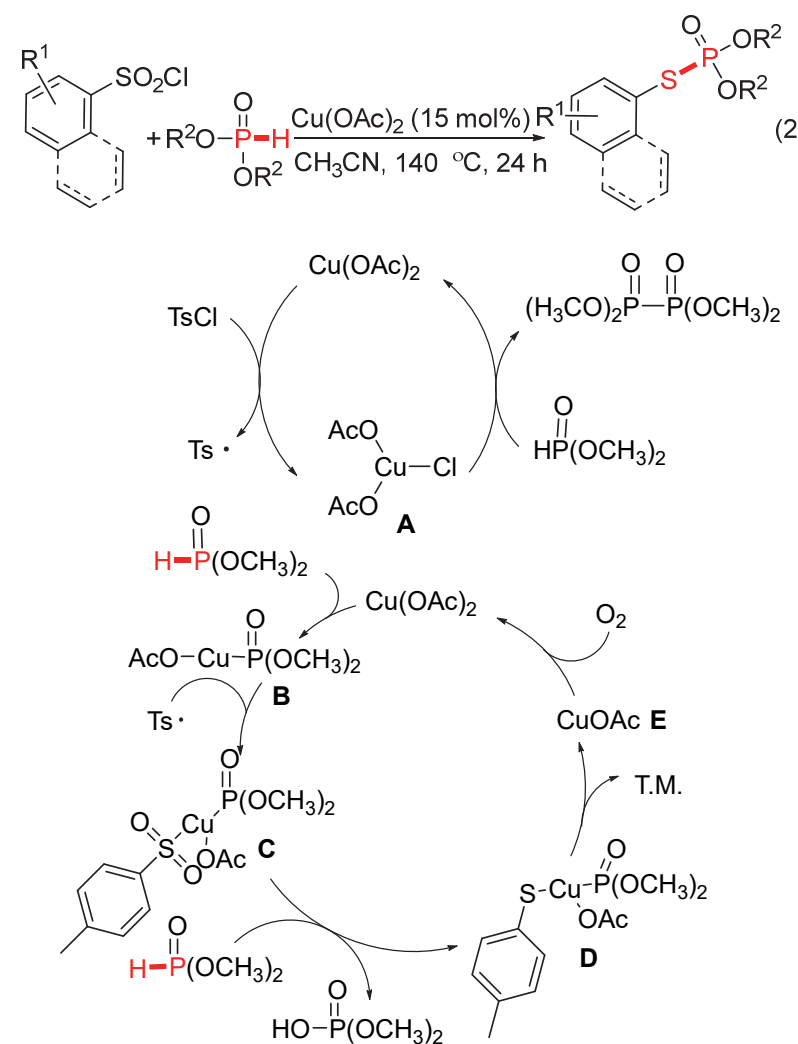

图式 $8 \mathrm{Cu}$ 催化芳基磺酰氯与烷氧基亚磷酸酯偶联制备 S-芳 基硫代膦酸酯

Scheme $8 \mathrm{Cu}$-catalyzed coupling of arylsulfonyl chloride with alkoxy phosphite to prepare S-aryl thiophosphonates

他们认为对甲苯磺酰氯与醋酸铜 $\left[\mathrm{Cu}(\mathrm{OAc})_{2}\right]$ 进行单 电子转移得到三价铜中间体 $\mathbf{A}$ : 然后与 $\mathrm{P}(\mathrm{O})-\mathrm{H}$ 化合物 之间进行配体交换反应, 得到中间体 $\mathbf{B}$ 并释放 HOAc. 中间体 $\mathbf{B}$ 被对甲苯磺酰基自由基进攻获得 $\mathrm{Cu}(\mathrm{III})$ 中间 体 $\mathbf{C}$; 再通过 $\mathrm{H}$-膦酸酯还原中间体 $\mathbf{C}$ 得到 $\mathrm{Cu}(\mathrm{III})$ 中间 体 $\mathbf{D}$; 最后一步还原消除得到目标产物 $S$-芳基硫代磷酸 酯, 并产生 $\mathrm{Cu}(\mathrm{I})$ 中间体 $\mathbf{E}$, 最后, $\mathbf{E}$ 被氧气氧化为 $\mathrm{Cu}(\mathrm{II})$, 继续进入下一循环中.

2017 年, 我们课题组 ${ }^{[34]}$ 发现在 $\mathrm{CuI}$ 与 $L$-proline 的协 同作用下也可以构建 $\mathrm{P}(\mathrm{O})-\mathrm{S}$ 键(Eq. 24), 该方法适用于 多种 $\mathrm{P}(\mathrm{O})-\mathrm{H}$ 结构, 并且可以实现催化循环 10 次仍能 以较好的产率得到目标化合物, 通过控制实验及机理的 研究发现该反应不需要氧气的参与. 最近, Moghaddam 等 ${ }^{[35}$ 利用可磁力回收的铁酸铜 $\left(\mathrm{CuFe}_{2} \mathrm{O}_{4}\right)$ 作为催化剂, 在无溶剂的条件下实现了磺酰氯与 $\mathrm{P}(\mathrm{O})-\mathrm{H}$ 试剂的偶 联(Eq. 25).

\section{2 非金属催化合成硫代磷(膦)酸酯}

2017 年, 我们课题组 ${ }^{[36]}$ 开发了在无金属、无碱条件

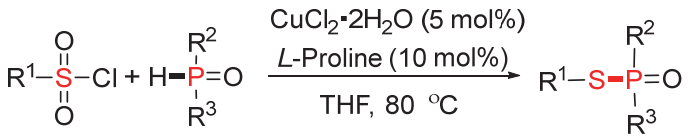

$$
\begin{aligned}
& \mathrm{R}^{1}=\text { alkyl, aryl } \\
& \mathrm{R}^{2}, \mathrm{R}^{3}=\text { O-alkyl, O-aryl, aryl } \\
& 27 \text { examples } \\
& \text { (52\% 90\%) }
\end{aligned}
$$

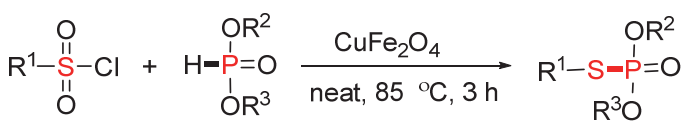

$$
\begin{aligned}
& \mathrm{R}^{1}=\text { ary } \\
& R^{2}, R^{3}=\text { O-alkyl }
\end{aligned}
$$

下 $\mathrm{P}(\mathrm{O})-\mathrm{H}$ 化合物与磺酰氯进行还原偶联反应构建 $\mathrm{P}(\mathrm{O})-\mathrm{S}$ 键的方法. 该方法条件温和, 反应时间较短, 产率最高可达 92\% (Eq. 26). 此外, 这种方法的底物拓 展性好, 对于芳基或烷基磺酰氯都取得了良好的收率. 但是对于 $\mathrm{P}$ 试剂有一定局限性.

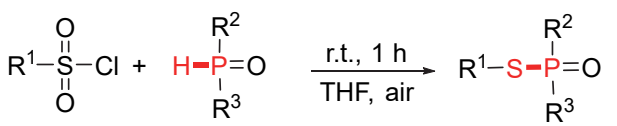

$$
\begin{aligned}
& \mathrm{R}^{1}=\text { alkyl, aryl } \\
& \mathrm{R}^{2}, \mathrm{R}^{3}=\text { aryl, O-aryl } \\
& \text { (32\% 92\%) }
\end{aligned}
$$

2017 年, Hong 等 ${ }^{[37]}$ 利用亚磺酸作为硫源，与 $\mathrm{P}(\mathrm{O})-\mathrm{H}$ 试剂在三苯基膦 $\left(\mathrm{PPh}_{3}\right)$ 调控下发生交叉偶联 (Eq. 27). 该反应条件温和, 不需要金属与氧化剂参与, 并且适用于各种 $P$ 试剂 $\left[P(O)-H, R_{3} P O\right]$, 对于手型磷试 剂, 可得到保持手性不变的产物.

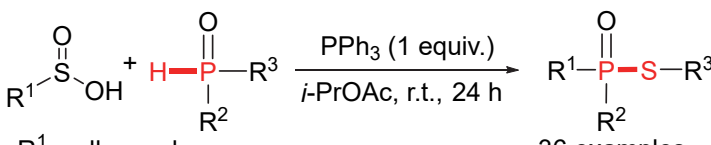

$$
\begin{aligned}
& \mathrm{R}^{1}=\text { alky, aryl } \\
& \mathrm{R}^{2}, \mathrm{R}^{3}=\text { O-alkyl, O-aryl, aryl, alky } \\
& 36 \text { examples } \\
& \text { (54\% 97\%) }
\end{aligned}
$$

作者对反应机理进行了研究，并提出了合理推测 (Scheme 9): 通过二苯基氧化膦互变异构得到的含磷中 间态，与亚磺酸脱水原位生成的亚砜基阳离子发生亲核 反应得到中间体 $\mathbf{A}$, 最后通过三苯基膦还原得到目标产 物, 另外一种推测是, 反应液中的亚磺酸可以的到硫代 亚硫酸盐或二硫化物，其可与发生互变异构的含磷中间 态发生取代得到目标产物.

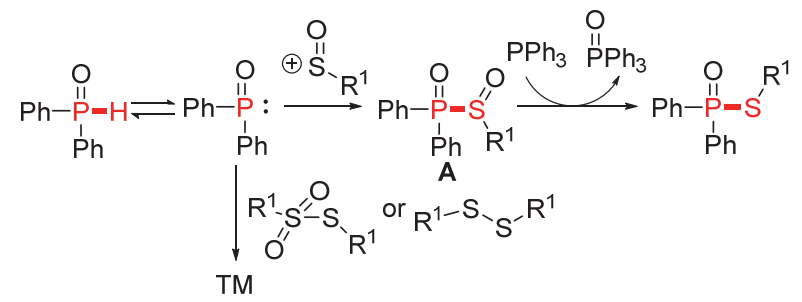

图式 $9 \mathrm{P}(\mathrm{O})$ - $\mathrm{H}$ 与磺酸直接偶联构建 $\mathrm{P}(\mathrm{O})-\mathrm{S}$ 键 Scheme 9 Metal- and oxidant-free $\mathrm{P}(\mathrm{O})-\mathrm{S}$ bond construction via direct coupling of $\mathrm{P}(\mathrm{O})-\mathrm{H}$ with sulfinic acids 
2017 年，易文斌等 ${ }^{[38]}$ 采用磺酸钠作为合成硫代磷 (膦)酸酯的硫源, 利用硫酸催化构建 $\mathrm{P}(\mathrm{O})-\mathrm{S}$ 键, 水作 为溶剂避免了有机溶剂的参与, 产率为 $12 \% \sim 81 \%$ (Eq. 28). 作者对反应机理进行了合理的推测, 认为该反应 为自由基反应.

$$
\begin{aligned}
& \mathrm{Ar}^{-\mathrm{S}_{-\mathrm{ONa}}^{\mathrm{O}}}+\underset{\mathrm{R}}{\mathrm{H}-\mathrm{P}_{\mathrm{P}}^{\mathrm{O}}-\mathrm{R}} \frac{\mathrm{H}_{2} \mathrm{SO}_{4}(0.25 \text { equiv. })}{\mathrm{H}_{2} \mathrm{O} \text {, r.t., } 6 \mathrm{~h}} \mathrm{Ar}-\mathrm{S}-\stackrel{\mathrm{P}}{\mathrm{P}}-\mathrm{R}_{\mathrm{R}}^{\mathrm{O}} \\
& \mathrm{R}=\text { aryl, O-alkyl, O-aryl }
\end{aligned}
$$

2019 年, 刘腾等 ${ }^{[39]}$ 开发了一种利用四丁基碘化铵 (TBAI)作为氧化剂在氧气存在下催化的氧化交叉偶联, 以磺酰肼为底物实现了硫代磷(膦)酸酯的高效合成(Eq. 29). 实验证明, 氧气在反应过程中起着重要作用, 当反 应在氮气条件下进行时基本不能得到产物. 同时, 该方 法可以实现克级制备, 并合成具有生物活性的分子.

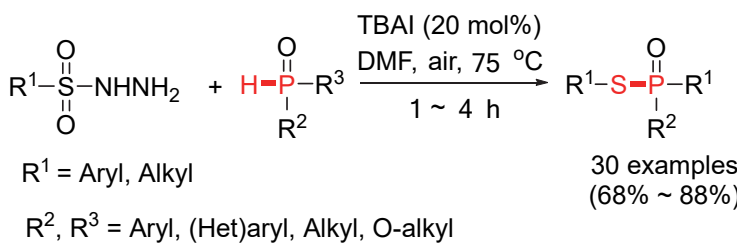

$$
\begin{aligned}
& \begin{array}{l}
\text { Inezin, } 68 \% \\
\text { gram-level, } 65 \%
\end{array} \\
& \begin{array}{l}
\text { An anticholinesterase, } 77 \% \\
\text { gram-level, } 76 \%
\end{array}
\end{aligned}
$$

\section{4 硫单质参与的多组分合成硫代磷(膦)酸酯}

硫单质因其价格低廉，在自然界简单易得，也广泛 用于硫代磷(膦)酸酯的合成.

\section{1 多组分分步合成硫代磷(膦)酸酯}

目前, 通过微波辐射的方法构建 $\mathrm{P}(\mathrm{O})$ - $\mathrm{S}$ 键是一种 高效的途径, 不仅反应时间短, 而且产物收率可观. 2002 年, Kaboudin 等 ${ }^{[40]}$ 利用微波辐射手段, 报道了一种 三组分合成硫代磷酸酯类化合物的反应(Eq. 30). 即在 无溶剂条件下, 亚磷酸二乙酯和硫粉通过醋酸铵 $\left(\mathrm{NH}_{4} \mathrm{OAc}\right)$ 和三氧化二铝 $\left(\mathrm{Al}_{2} \mathrm{O}_{3}\right)$ 的协同作用, 继续与烷 基卤代物反应得到相应的硫代磷酸酯. 在此基础上, 2006 年, Kaboudin 等 ${ }^{[4]}$ 对该反应体系进一步研究与改 进, 以三乙胺 $\left(\mathrm{Et}_{3} \mathrm{~N}\right)$ 代替醋酸铵 $\left(\mathrm{NH}_{4} \mathrm{OAc}\right)$, 在微波条件 下成功实现了 $\mathrm{P}(\mathrm{O})-\mathrm{H}$ 化合物到硫代磷酸酯的转变 $(\mathrm{Eq}$. 31).

\section{2 三组分一锅法合成硫代磷(膦)酸酯}

从上述的文献报道中可以看出, 目前在利用有机 磷、硫化合物相互作用合成硫代磷(膦)酸酯的方法上尚

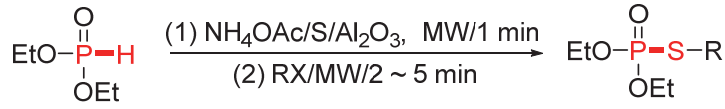

$$
\begin{aligned}
& \mathrm{R}=\text { alkyl, } \mathrm{X}=\mathrm{Br}^{-}, \mathrm{Cl}^{-} \\
& 10 \text { examples } \\
& \text { (75\% 90\%) }
\end{aligned}
$$

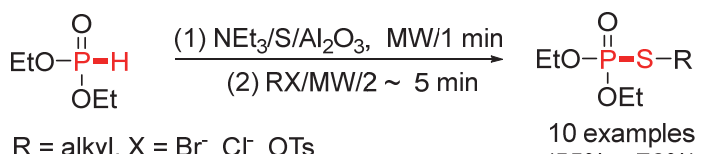

$$
\begin{aligned}
& \text { (55\% 76\%) }
\end{aligned}
$$

有不足之处，如底物多是二组分协同、反应分步、能耗 高等. 因此，改用操作简单的 “一锅法” 制备硫代磷(膦) 酸酯具有广阔的前景.

与微波辐射的方法相比，以路易斯酸催化 $\mathrm{P}(\mathrm{O})-\mathrm{H}$ 化合物的偶联反应同样具有反应时间短，产物收率高等 优点. 2006 年，施敏等 ${ }^{[42]}$ 在路易斯酸三氟甲磺酸锡 $\left[\mathrm{Sn}(\mathrm{OTf})_{2}\right]$ 催化的亚甲基环丙烷衍生物、硫(硒)与二苯基 氧膦三组分的偶联反应中, 利用开环反应，以良好的产 率实现了二芳基硫(硒)代磷酸酯类化合物的合成(Eq. $32)$.

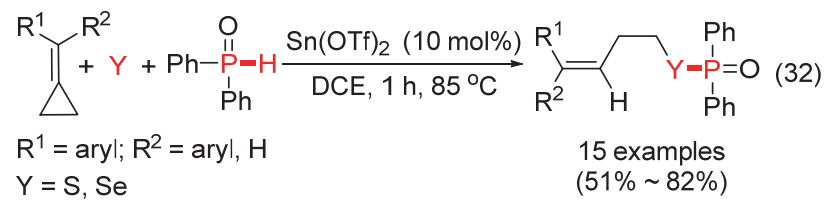

作者对反应机理进行了合理推测(Scheme 10): $\mathrm{Y}$ 单 质与二苯基膦反应生成 $\mathrm{Ph}_{2} \mathrm{P}(\mathrm{Y}) \mathrm{OH}$, 通过相互转换得到 $\mathrm{Ph}_{2} \mathrm{P}(\mathrm{O}) \mathrm{YH}$, 最后与 $\mathrm{Sn}(\mathrm{OTf})_{2}$ 活化的亚甲基环丙烷类衍 生物(中间体 $\mathbf{A}$ )反应，得到相应的开环产物.

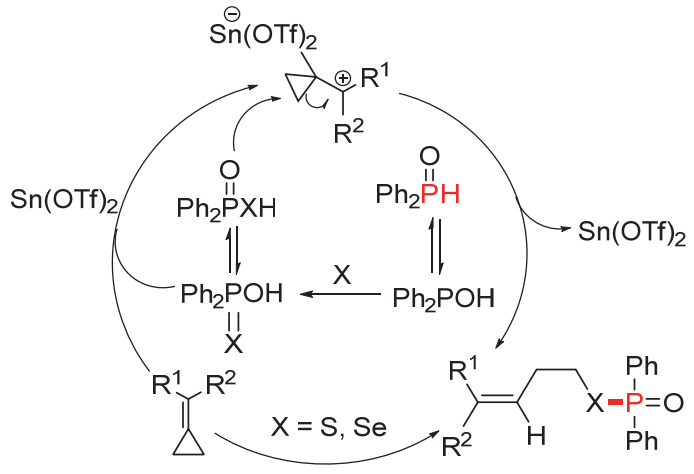

图式 10 Sn 催化制备硫(硒)代膦酸酯

Scheme 10 Preparation of sulfur (selenium) phosphonate by $\mathrm{Sn}$ catalysis

赵玉芬课题组 ${ }^{[43]}$ 在 2016 年报道了芳基硼酸、硫单 质与烷氧基亚磷酸酯化合物三组分 “一锅法” 合成硫代 磷酸酯的反应(Eq. 33). 该反应中, 三氟甲磺酸铜作为催 化剂与双齿氮配体联吡啶协同作用，以较优异的收率制 备一系列 S-芳基硫代磷酸酯. 此外，该方法也适用于克 级规模的合成. 


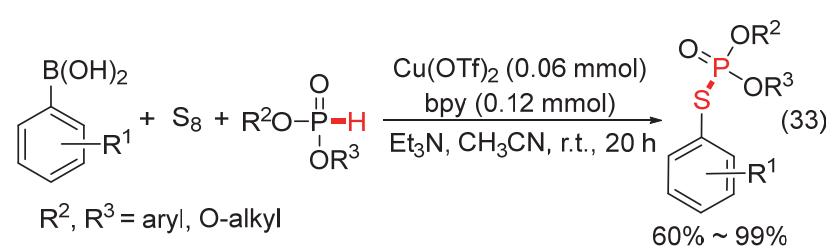

在此基础上, 赵玉芬等 ${ }^{[44]}$ 再次报道了两种不同的 铜催化体系下多组分 “一锅法” 制备 S-芳基硫代磷酸酯 的新方法. 方法一是: 采用芳基化试剂二芳基碘鎓盐为 反应物, 与硫粉、 $\mathrm{P}(\mathrm{O})-\mathrm{H}$ 化合物三组分协同作用, 在 乙酰丙酮酸铜的催化作用下, 利用偶联反应构建 $\mathrm{P}(\mathrm{O})$ $\mathrm{S}-\mathrm{C}$ 键(Eq. 34); 方法二是: 利用芳基重氮盐与硫粉、 $\mathrm{P}(\mathrm{O})-\mathrm{H}$ 化合物三组分在三氟甲磺酸铜的催化下进行反 应(Eq. 35). 以上两种方法均能以优良的产率得到相应 的目标产物, 同时, 反应底物具有良好的普适性且官团 能耐受性强, 烷氧基亚磷酸酯以及芳基氧膦类化合物也 适用于该反应.

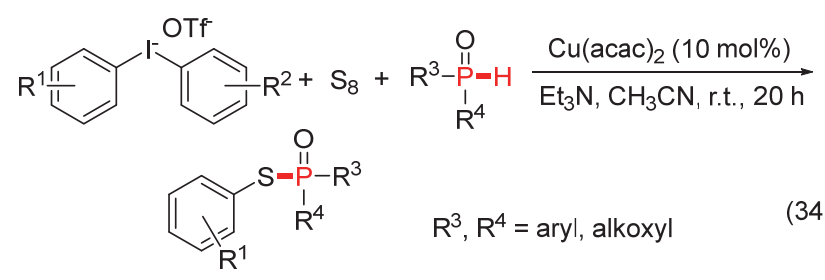
$65 \% \sim 95 \%$

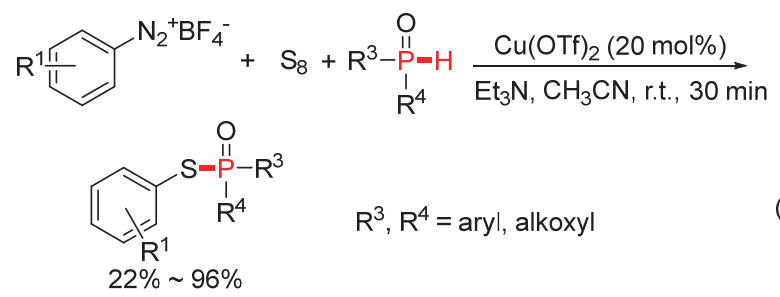

2017 年, 本课题组 ${ }^{[45]}$ 开发了伯烷基卤化物、硫单质 和 $\mathrm{P}(\mathrm{O})-\mathrm{H}$ 化合物三组分“一锅法”的偶联反应(Eq. 36). 在三乙胺的促进下, 以水代替有机溶剂作为反应介质, 高效地实现了 $\mathrm{P}(\mathrm{O})-\mathrm{S}-\mathrm{C}$ 烷基化的转变. 该反应的优 势在于底物拓展性强、产率较高, 但是值得注意的是, 当反应时间过长, 在过量有机碱作用下会对反应有一定 的影响.

2017 年, 张致慧等 ${ }^{[46]}$ 报道了一种利用磁性可回收 的 MOF 材料 $\left(\mathrm{Cu}-\mathrm{BTC} @ \mathrm{Fe}_{3} \mathrm{O}_{4}\right)$ 催化苯胺、硫粉、磷试剂 三组分的反应(Eq. 37). 该方法适用于多种磷试剂, 并且 该材料可以催化循环 6 次仍以 $80 \%$ 的收率得到目标化合 物.

2020 年, 赵玉芬等 ${ }^{[47]}$ 组利用三组分一锅法, 在铜催 化下首次实现了包含磺酰胺或酰胺远端烷基(Eq. 38)以 及苠基的硫代磷酸酯的合成(Eq. 39) 该方法极大地拓展

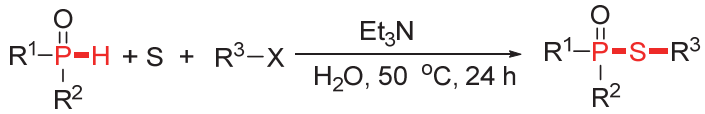

$$
\begin{aligned}
& \mathrm{R}^{1}, \mathrm{R}^{2}=\text { aryl, O-aryl, O-alkyl; } \mathrm{R}^{3}=\text { alkyl; } \mathrm{X}=\mathrm{Cl}, \mathrm{Br}, \mathrm{I}, \mathrm{OTs}
\end{aligned}
$$<smiles>C#CCSP(=O)(Oc1ccccc1)c1ccccc1</smiles>

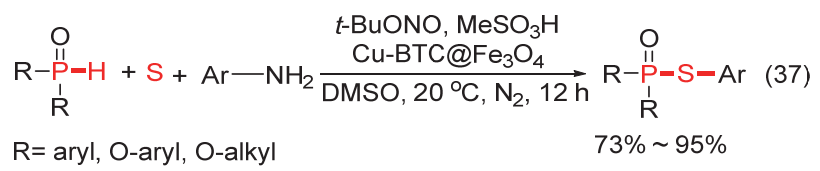

了硫代磷酸酯的反应底物类别. 通过对照实验推测该反 应经历了 1,5-HATP 转变的自由基历程.
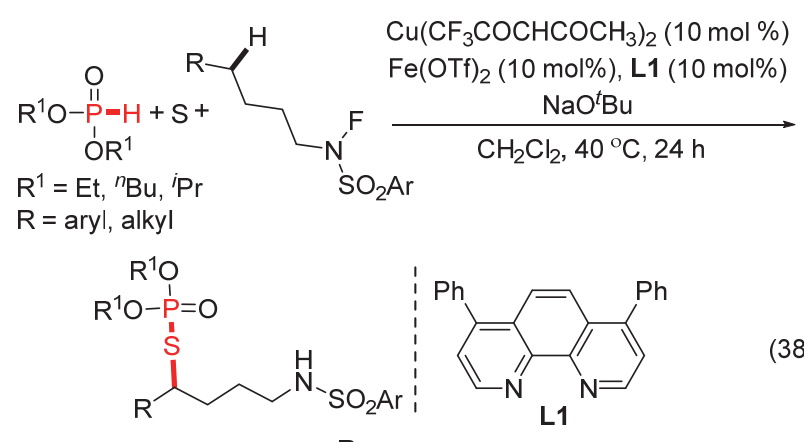

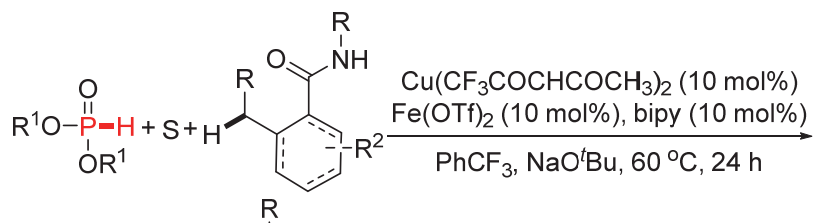

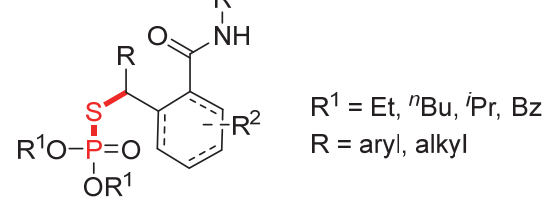

\section{5 其他}

2017 年, 吴劼等 ${ }^{[48]}$ 采用 $\mathrm{DABCO} \cdot\left(\mathrm{SO}_{2}\right)_{2}$ 作为硫源, 与四氟化嗍碘代芳基化合物、芳基 $\mathrm{P}(\mathrm{O})-\mathrm{H}$ 试剂三组分 在三(2-苯基吡啶)合铱 $\left[\operatorname{Ir}(\mathrm{ppy})_{3}\right]$ 催化的可见光作用下, 以较好的产率实现了硫代膦酸酯的合成. 通过机理的研 究发现, 该反应在可见光照射下利用 $\operatorname{Ir}(\mathrm{III})$ 到 $\operatorname{Ir}(\mathrm{V})$ 的循 环来实现催化(Eq. 40), 产率可达 46\% 91\%.

2019 年, Saha 等 ${ }^{[49]}$ 报道了室温条件下, $\mathrm{P}(\mathrm{O})-\mathrm{H}$ 化 合物与 $N$-硫族二甲酰亚胺反应直接获得硫代磷(膦)酸 


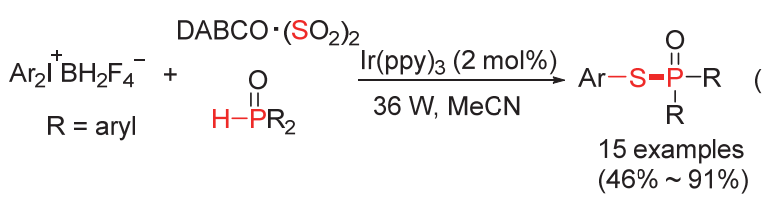

酯的方法(Eq. 41). 该方法避免了有机溶剂、催化剂、碱 以及添加剂的使用, 具有反应条件温和, 操作简便等特 点, 并且对于硒代磷酸酯类化合物的合成也同样适用.

$$
\begin{aligned}
& \mathrm{R}^{1}-\mathrm{S}-\mathrm{N} \\
& \mathrm{R}^{1}=\text { Aryl, Alkyl } \\
& \mathrm{R}^{2}=\text { Aryl, O-alkyl } \\
& 15 \text { examples } \\
& (83 \% \sim 91 \%)
\end{aligned}
$$

利用交叉偶联构建 $\mathrm{P}(\mathrm{O})-\mathrm{S}$ 键大多得到的是芳基硫 代磷酸酯, 对于烷基硫代磷酸酯或者构建较为复杂含有 手性磷结构的硫代磷酸酯报道较少. 由 $\mathrm{P}(\mathrm{O})-\mathrm{H}$ 试剂出 发先得到含有 $\mathrm{P}(\mathrm{O})-\mathrm{S}$ 结构的中间体, 再与其他试剂构 建 $\mathrm{S}-\mathrm{C}$ 键也可以合成硫代磷酸酯. 2010 年, $\mathrm{Wu}$ 课题 组 ${ }^{[50]}$ 利用烯丙基醇或者烯丙基醚与硫代磷酸 $(\mathrm{P}(\mathrm{O})-\mathrm{SH}$ 试剂)作用合成了一系列硫代磷酸酯(Eq. 42), 但是该反 应只适用于 $\gamma, \gamma$-二取代的烯基醇，对于脂肪族的一级、 二级、三级醇以及芳基醇不适用，随后，他们 ${ }^{[51]}$ 利用 $\mathrm{Ga}(\mathrm{OTf})_{3}$ 催化醇类与 $\mathrm{P}(\mathrm{O})-\mathrm{SH}$ 试剂, 克服了上述缺点 (Eq. 43).

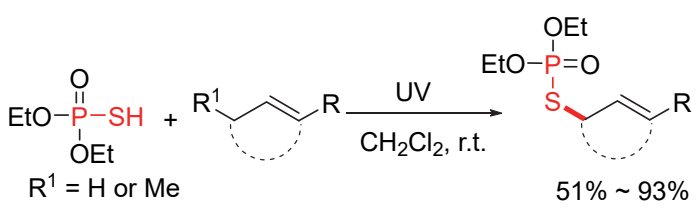

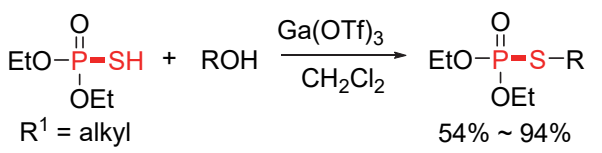

采用 $\mathrm{P}(\mathrm{O})-\mathrm{H}$ 试剂直接构建 $\mathrm{P}(\mathrm{O})-\mathrm{S}$ 键来合成具有 手性结构的方法比较受限, 2019 年 Schoenebeck 小组 ${ }^{[52]}$ 利用在空气中较为稳定的 $\operatorname{Pd}(\mathrm{I})$ 二聚体催化硫代磷酸盐 与芳基碘代物，不仅拓展了磷试剂的种类，还合成了一 系类具有手性的芳基硫代磷酸酯(Eq. 44). 特别的是, 该 方法实现了直接合成 TADDOL 骨架的手性芳基硫代磷 酸酯. 不仅产率高而且对映选择性 $>99 \%$ ee. 该方法为 在合成具有手性分子的药物研究方面提供了重要的思 路.

在拓展烷基硫代磷酸酯的结构方面，2020 年, O'Sullivan 小组 ${ }^{[53]}$ 利用 $\mathrm{P}(\mathrm{O})-\mathrm{H}$ 与双硫代乙基氰形成的 含硫中间体，与 DBU 在室温下得到具有 DBU 结构的硫 代磷酸盐, 可以与多种烷基碘代物或烷基溴代物生成烷 基硫代磷酸酯, 弥补了直接构建 $\mathrm{P}(\mathrm{O})-\mathrm{S}$ 键不能得到复
杂烷基硫代磷酸酯结构的短板(Eq. 45).
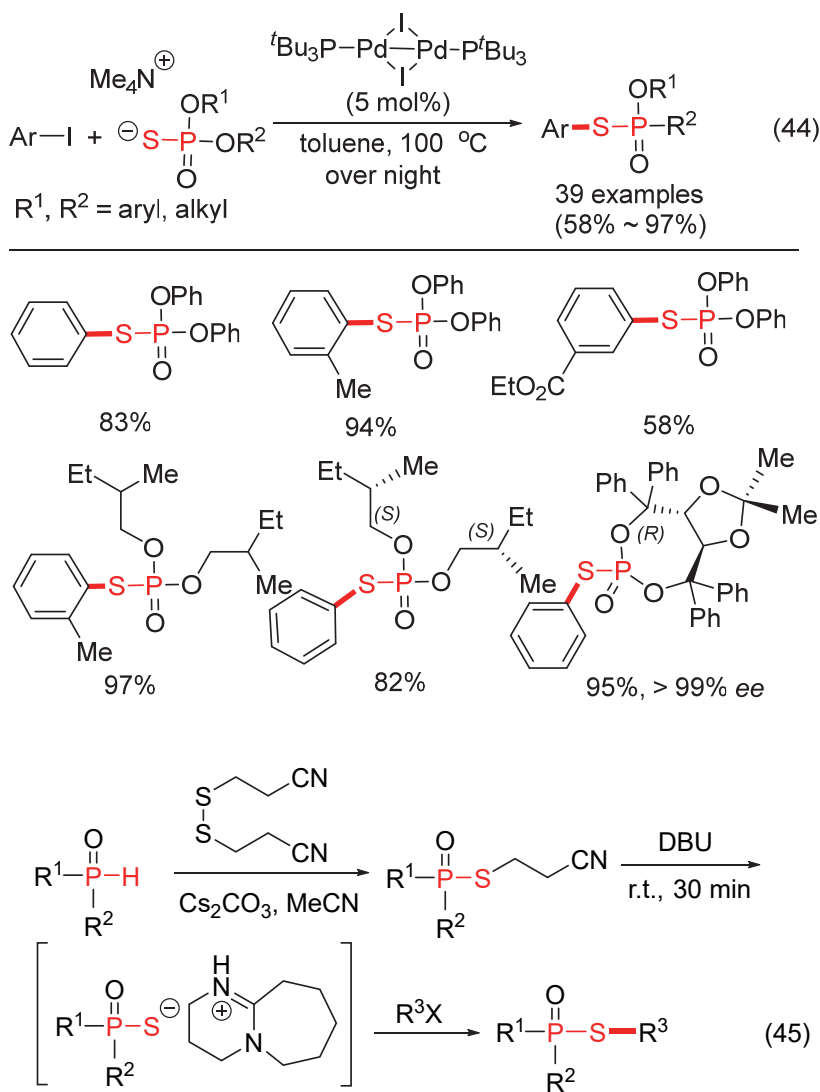

$\mathrm{R}^{1}, \mathrm{R}^{2}=$ O-alkyl, O-aryl, aryl; $\quad 35$ examples $\mathrm{R}^{3}=$ alkyl; $\mathrm{X}=\mathrm{Br}, \mathrm{I} \quad(71 \% \sim 91 \%)$
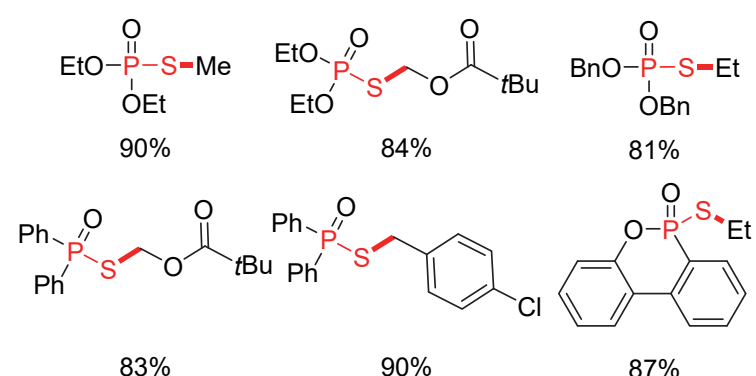

\section{6 总结}

综上所述，按照与 $\mathrm{P}(\mathrm{O})$ - $\mathrm{H}$ 化合物反应的含硫试剂 进行分类，综述了近年来合成硫代磷(膦)酸酯的方法. 目前常见的含硫试剂主要包括硫醇(酚)、双硫试剂、磺 酰类试剂、硫单质以及其他含硫试剂. 合成方法由过渡 金属催化逐步过渡到非金属催化以及光电催化, $\mathrm{P}(\mathrm{O})-$ $\mathrm{H}$ 种类从普通磷酸到多种手性结构. 硫代磷(膦)酸酯的 合成经历了质的变化.

尽管合成该类化合物的方法已取得重大进展，但高 效合成结构多样的含有 $\mathrm{P}(\mathrm{O})-\mathrm{S}-\mathrm{C}$ 键的化合物仍是未 来有机合成和金属催化化学领域的研究热点. 随着科技 的进步及人们环保意识的不断提升，绿色有机合成技术 
将越发受到重视. 开发原子经济性好, 利用率高, 污染 少的新方法来代替原有的污染大、经济性差的传统合成 方法迫在眉睫, 这对于构建 $\mathrm{P}(\mathrm{O})-\mathrm{S}-\mathrm{C}$ 键的研究具有 重要的指导意义, 也是未来合成有机磷/硫化合物的重 要研究方向.

\section{References}

[1] (a) Rhee, S. W.; Iyer, R. P.; Coughlin, J. E.; Padmanabhan, S.; Malerich, J. P.; Tanga, M. J. J. Labelled Compd. Radiopharm. 2012, $55,197$.

(b) Leisvuoria, A.; Ahmeda, Z.; Ora, M.; Beigelmanb, L.; Blattb, L.; Harri, L. Helv. Chim. Acta 2012, 95, 1512.

(c) Crooke, S. T.; Bennett, C. F. Annu. Rev. Pharmacol. Toxicol. 1996, 36, 107.

(d) Xie, R.-L.; Zhao, Q.-F.; Zhang, T.; Fang, J.; Mei, X.-D.; Ning, J.; Tang, Y. Bioorg. Med. Chem. 2013, 21, 278.

(e) Guga, P.; Boczkowska, M.; Janicka, M.; Maciaszek, A.; Nawrot, B.; Antoszczyk, S.; Stec, W. J. Pure Appl. Chem. 2006, 78, 993.

(f) Yan, H.-B.; Wang, X.-L.; KuoLee, R.; Chen, W.-X. Bioorg. Med. Chem. Lett. 2008, 18, 5631.

[2] (a) Jaiswal, A. K.; Rao, G. P.; Pandey, O. P.; Sengupta, S. K. J. Agric. Food Chem. 1998, 46, 1609.

(b) Myers, D. K.; Mendel, B.; Gersmann, H. R.; Ketelaar, J. A. Nature 1952, 170, 805 .

[3] (a) Fukuoka, M.; Shuto, S.; Minakawa, N.; Ueno; Y.; Matsuda, A. J. Org. Chem. 2000, 65, 5238.

(b) Jhajharia, P.; Samota, M. K.; Seth, G. Heteroat. Chem. 2010, 21, 84.

(c) Lauer, A. M.; Mahmud, F.; Wu, J. J. Am. Chem. Soc. 2011, 133, 9119 .

(d) Robertson, F.; Wu, J. Org. Lett. 2010, 12, 2668

(e) Lauer, A. M.; Wu, J. Org. Lett. 2012, 14, 5138.

[4] Bennett, M.; Macdonald, K.; Chan, S. W.; Luzio, J. P.; Simari, R. Science 1998, 282, 290.

[5] Gabelt, B.T.; Hennes, E. A.; Seeman, J.; Tian, B. Invest. Opth. Vis. Sci. 2004, 45, 2732.

[6] Kumar, T. S.; Yang, T. H.; Mishra, S.; Cronin, C.; Chakraborty, S.; Shen, J.-B.; Liang, B. T.; Jacobson, K. A. J. Med. Chem. 2013, 56, 902.

[7] Murdock, L. L.; Hopkins, T. L. J. Agric. Food Chem. 1968, 16, 954.

[8] Ishizaki, H.; Kobayashi, I.; Kunoh, H. Pestic. Sci. 1986, 17, 517. Kim, Y. S.; Kim, K. D. Crop. Prot. 2009, 28, 940.

[9] Michaelis-Arbuov: Hoffmann, F.; Moore, T.; Kagan, B. J. Am. Chem. Soc. 1956, 78, 6413.

[10] Xiong, B.; Zhou, Y.; Zhao, C.; Goto, M.; Yin, S.-F.; Han, L.-B. Tetrahedron 2013, 69, 9373.

[11] Wang G.; Shen, R.; Xu, Q.; Goto, M.; Zhao, Y.-F.; Han, L.-B. T. J. Org. Chem. 2010, 75, 3890.

[12] Kaboudin, B.; Abedi, Y.; Kato, J. Y.; Yokomatsu, T. Synthesis 2013, 45, 2323.

[13] Zhu, Y.-Y.; Chen, T.-Q.; Li, S.; Shimada, S.; Han, L.-B. J. Am. Chem. Soc. 2016, 138,5825 .

[14] Huang, H.; Ash, J.; Kang, J.-Y. Org. Biomol. Chem. 2018, 16, 4236.

[15] Li, S.; Chen, T.-Q.; Saga, Y. T.; Han, L.-B. RSC Adv. 2015, 5, 71544.

[16] Song, S.; Zhang, Y.-Q.; Yeerlan, A.; Zhang, B.-C.; Liu, J.-Z.; Jiao, N. Angew. Chem., Int. Ed. 2017, 56, 2487.

[17] He, W.; Hou, X.; Li, X.-J.; Song, L.; Yu, Q.; Wang, Z.-W. Tetrahe- dron 2017, 73, 3133

[18] Wang, J.-C.; Huang, X.; Ni, Z.-Q.; Wang, S.-C.; Wu, J.; Pan, Y.-J. Green. Chem. 2015, 17,314.

[19] Wang, J.-C.; Huang, X.; Ni, Z.-Q.; Wang, S.-C.; Pan, Y.-J.; Wu, J. Tetrahedron 2015, 71,7853.

[20] Bi, X.-J.; Li, J.-C.; Meng, F.-H.; Wang, H.-M.; Xiao, J.-H. Tetrahedron 2016, 72, 706.

[21] Sun, J.-G.; Weng, W.-Z.; Li, P.; Zhang, B. Green Chem. 2017, 19, 1128.

[22] Sun, J.-G.; Yang, H.; Li, P.; Zhang, B. Org. Lett. 2016, 18, 5114.

[23] Zhang, H.; Zhan, Z.; Lin, Y.; Shi, Y.-S.; Li, G.-B.; Wang, Q.-T.; Deng, Y.; Hai, L.; Wu, Y. Org. Chem. Front. 2018, 5, 1416.

[24] Li, Y.-J.; Yang, Q.; Yang, L.-Q.; Lei, N.; Zheng, K. Chem. Commun. 2019, 55, 4981.

[25] Torii, S.; Tanaka, H.; Sayo, N. J. Org. Chem. 1979, 44, 2938.

[26] Gao, Y.-X.; Tang, G.; Cao, Y.; Zhao, Y.-F. Synthesis 2009, 1081

[27] Ouyang, Y.-J.; Li, Y.-Y.; Li, N.-B.; Xu, X.-H. Chin. Chem. Lett. 2013, 24, 1103.

[28] Ouyang, Y.-J.; Q, R.-H.; Chen, S.-H.; Chen, J.-Y.; Xu, X.-H. Chin. J. Org. Chem. 2015, 35, 2636.

[29] Wang, J.-X.; Wang, X.-L.; Li, H.-J.; Yan, J. J. Organomet. Chem. 2018, 859,75 .

[30] Choudhary, R.; Singh, P.; Rekha, B.; Sharma, M. C.; Badsara, S. S. Org. Biomol. Chem. 2019, 17, 9757.

[31] Kumaraswamy, G.; Raju, R. Adv. Synth. Catal. 2014, 356, 2591.

[32] Bai, J.; Cui, X.-L.; Wang, H.; Wu, Y.-J. Chem. Commun. 2014, 50, 8860.

[33] Zhang, X.-H.; Wang, D.-G.; An, D.; Han, B.-S.; Song, X.; Li, L.; Zhang, G.-Q; Wang, L.-X. J. Org. Chem. 2018, 83, 1532.

[34] Moghaddam, F. M.; Daneshfar. M.; Azaryan, R. Phosphorus Sulfur Relat. Elem. 2020, 196, 311

[35] Wang, D.-G; Zhao, J.-L; Xu, W.-G.; Shao, C.-W.; Shi, Z.; Li, L.; Zhang, X.-H. Org. Biomol. Chem. 2017, 15, 545.

[36] Moon, Y.; Moon, Y.; Choi, H.; Hong, S. Green Chem. 2017, 19, 1005.

[37] Lin, Y.-M.; Lu, G.-P.; Wang, G.-X.; Yi, W.-B. J. Org. Chem. 2017, $82,382$.

[38] Liu, T.; Zhang, Y, Q.; Yu, R.; Liu, J, J.; Cheng, F, X. Synthesis 2020, $52,253$.

[39] Kaboudin, B. Tetrahedron Lett. 2002, 43, 8713.

[40] Kaboudin, B.; Farjadian, F. Beilstein J. Org. Chem. 2006, 2,4

[41] Shi, M.; Jiang, M.; Liu, L.-P. Org. Biomol. Chem. 2007, 5, 438.

[42] Xu, J.; Zhang, L.-L.; Li, X.-Q.; Gao, Y.-Z.; Tang, G.; Zhao, Y.-F. Org. Lett. 2016, 18, 1266.

[43] Zhang, L.-L.; Zhang, P.-B.; Li, X.-Q.; Xu, J.; Tang, G.; Zhao, Y.-F J. Org. Chem. 2016, 81, 5588.

[44] Zhang, X.-H.; Shi, Z.; Shao, C.-W.; Zhao, J.-L.; Wang, D.-G.; Zhang, G.-Q.; Li, L. Eur. J. Org. Chem. 2017, 14, 1884.

[45] Wang, L.; Yang, S.; Chen, L.; Yuan, S.; Chen, Q.; He, M.-Y.; Zhang, Z.-H. Catal. Sci. Technol. 2017, 7, 2356.

[46] Shi, S.-S.; Zhang, P.-B.; Luo, C.; Zhuo, S.-H.; Zhang, Y.-M.; Tang, G.; Zhao, Y. F. Org. Lett. 2020, 22,1760.

[47] Gong, X.-X.; Chen, J.-H.; Liu, J.-H.; Wu, J. Org. Chem. Front. 2017, 4, 2221.

[48] Mondal, M.; Saha, A. Tetrahedron Lett. 2019, 60, 150965.

[49] Han, X.-P.; Zhang, Y.-H.; Wu, J. J. Am. Chem. Soc. 2010, 132, 4104.

[50] Han, X.-P, Wu, J. Org. Lett. 2010, 24, 5780.

[51] Chen, X.-Y.; Pu, M.; Cheng, H.-G.; Sperger, T.; Schoenebeck, F. Angew. Chem., Int. Ed. 2019, 58, 11395.

[52] Jones, D. J.; O'Leary, E. M.; O'Sullivan, T. P. Adv. Synth. Catal. 2020, 362, 1825 . 